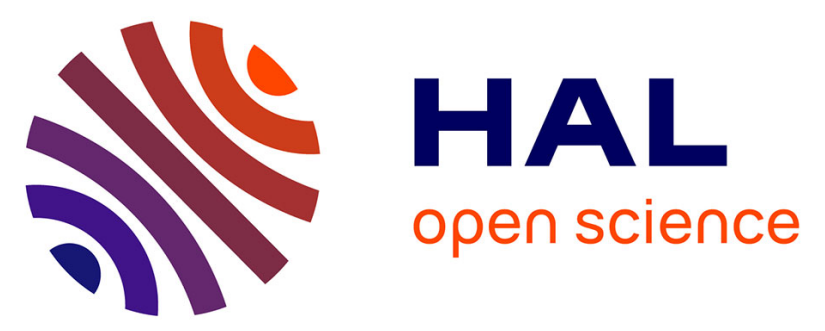

\title{
RhoA GTPase regulates radiation-induced alterations in endothelial cell adhesion and migration
}

\author{
Matthieu Rousseau, Marie-Hélène Gaugler, Audrey Rodallec, Stéphanie \\ Bonnaud, François Paris, Isabelle Corre
}

\section{- To cite this version:}

Matthieu Rousseau, Marie-Hélène Gaugler, Audrey Rodallec, Stéphanie Bonnaud, François Paris, et al.. RhoA GTPase regulates radiation-induced alterations in endothelial cell adhesion and migration. Biochemical and Biophysical Research Communications, 2011, 414 (4), pp.750 - 755. 10.1016/j.bbrc.2011.09.150 . hal-03522644

\section{HAL Id: hal-03522644 \\ https://hal.science/hal-03522644}

Submitted on 12 Jan 2022

HAL is a multi-disciplinary open access archive for the deposit and dissemination of scientific research documents, whether they are published or not. The documents may come from teaching and research institutions in France or abroad, or from public or private research centers.
L'archive ouverte pluridisciplinaire HAL, est destinée au dépôt et à la diffusion de documents scientifiques de niveau recherche, publiés ou non, émanant des établissements d'enseignement et de recherche français ou étrangers, des laboratoires publics ou privés.

\section{(1) (1) $\$$}

Distributed under a Creative Commons Attribution - NonCommercial - NoDerivatives 44.0 


\title{
RhoA GTPase regulates radiation-induced alterations in endothelial cell adhesion and migration
}

\author{
Matthieu Rousseau ${ }^{1}$, Marie-Hélène Gaugler, Audrey Rodallec, Stéphanie Bonnaud, François Paris, \\ Isabelle Corre* \\ Inserm UMR U892, Centre de Recherche en Cancérologie Nantes-Angers CRCNA, Institut de Recherche Thérapeutique IRT-UN, Université de Nantes, 8 Quai Moncousu, BP 70721, \\ F-44007, France
}

\section{A R T I C L E I N F O}

\section{Article history:}

Received 28 September 2011

Available online 6 October 2011

\section{Keywords:}

RhoA

Endothelial cells

Ionizing radiation

Cytoskeleton

Adhesion

Migration

\begin{abstract}
A B S T R A C T
Endothelial cells of the microvasculature are major target of ionizing radiation, responsible of the radiationinduced vascular early dysfunctions. Molecular signaling pathways involved in endothelial responses to ionizing radiation, despite being increasingly investigated, still need precise characterization. Small GTPase RhoA and its effector ROCK are crucial signaling molecules involved in many endothelial cellular functions. Recent studies identified implication of RhoA/ROCK in radiation-induced increase in endothelial permeability but other endothelial functions altered by radiation might also require RhoA proteins. Human microvascular endothelial cells HMEC-1, either treated with Y-27632 (inhibitor of ROCK) or invalidated for RhoA by RNA interference were exposed to $15 \mathrm{~Gy}$. We showed a rapid radiation-induced activation of RhoA, leading to a deep reorganisation of actin cytoskeleton with rapid formation of stress fibers. Endothelial early apoptosis induced by ionizing radiation was not affected by Y-27632 pre-treatment or RhoA depletion. Endothelial adhesion to fibronectin and formation of focal adhesions increased in response to radiation in a RhoA/ROCK-dependent manner. Consistent with its pro-adhesive role, ionizing radiation also decreased endothelial cells migration and RhoA was required for this inhibition. These results highlight the role of RhoA GTPase in ionizing radiation-induced deregulation of essential endothelial functions linked to actin cytoskeleton.
\end{abstract}

(c) 2011 Elsevier Inc. All rights reserved.

\section{Introduction}

Exposure to ionizing radiation (IR), either accidental (i.e. nuclear power plant incident) or used as therapeutical strategy in radiotherapy cancer treatment, leads to severe acute damage to normal human tissues. Microvasculature, as an ubiquitous organ system, is known to play a major role in the pathogenesis of radiationinduced injury to normal tissues [1]. Endothelial cells (EC) that form the inner lining of microvessels, are critical targets for IR and are responsible of radiation-induced acute vascular dysfunctions [2]. Apoptotic cell death, altered cell morphology, modulation of biomolecule synthesis and expression but also perturbation of barrier function are among the immediate responses of the endothelial compartment to IR. Molecular signaling involved in these early

\footnotetext{
* Corresponding author. Address: Inserm UMR U892, Centre de Recherche en Cancérologie Nantes-Angers CRCNA, Institut de Recherche Thérapeutique UN, 8 Quai Moncousu, BP7072, 44007 Nantes Cedex 1, France. Fax: +33 (0)228 080204. E-mail address: icorre@nantes.inserm.fr (I. Corre)

1 Present address: Centre de Recherche en Rhumatologie et Immunologie, Centre de Recherche du Centre Hospitalier Universitaire de Québec, Faculté de Médecine de l'Université Laval, Québec, Canada G1V 4G2.
}

endothelial responses to radiation appeared to involve GTPase RhoA as increased vascular permeability induced by IR was recently shown to be orchestrated by this protein [3].

The Rho family GTPases, RhoA, Rac-1 and Cdc42 are membranelinked molecular switches, initially characterized as playing a pivotal role in regulating the actin cytoskeleton but also involved in a wide array of essential cellular processes [4]. In the endothelial compartment, RhoA and its major effector Rho-associated kinase (ROCK) are key signaling molecules activated in response to a variety of stimuli (vasoactive substances, shear stress, pro-angiogenic factors, oxidative stress) and RhoA/ROCK pathway regulates numerous endothelial cellular functions like permeability, migration and adhesion [5], but also cell survival and apoptosis [6,7]. Nevertheless, implication of the Rho family GTPases in endothelial cellular responses to IR remains poorly described. One study, addressing the role of RhoA in normal microvasculature exposed to single-high dose of IR, demonstrated recently that radiation-induced actin cytoskeleton reorganization and increased permeability by modification of VE-cadherin adherens junctions was mediated by RhoA proteins [3]. Increased permeability is one of the acute responses of normal endothelium to radiation leading to vascular injury but other functions linked to RhoA may also be altered by IR stress in normal 
endothelium. It has already been described but in a tumoral and in angiogenic context that RhoA was linked to IR-induced increase in endothelial adhesion to tumor cells and to radioresistant brain EC migration, respectively [8,9]. In this study, we aimed at characterizing the role of RhoA signaling protein in early endothelial functions rapidly affected by IR in normal microvascular endothelial cells.

\section{Materials and methods}

\subsection{Reagents}

Fibronectin, Phalloidin-TRITC (Tetramethylrhodamine B Isothiocyanate) were from Sigma-Aldrich (St Quentin, Fr). Rho-associated protein kinase (ROCK) inhibitor Y-27632 [10] was from Calbiochem (Nottingham, UK). The following anti-human antibodies were used: RhoA (sc-418) and actin (Santa Cruz Biotechnology, CA, USA), vinculin (hVIN-1) (Sigma-Aldrich). Secondary antibodies Alexa Fluor ${ }^{\circledR} 568$ or 647 goat anti-mouse Ig were from Molecular Probes (Cergy-Pontoise, Fr).

\subsection{Cell culture and irradiation}

Human microvascular endothelial cells (HMEC-1) were cultured as described [11]. Subconfluent HMEC-1 were serum-starved for $18 \mathrm{~h}$, before irradiation at $15 \mathrm{~Gy}$ delivered at a rate of $1.28 \mathrm{~Gy} /$ min (Faxitron CP160 irradiator, Faxitron X-ray Corporation, IL, USA).

\subsection{Apoptosis detection}

Apo2.7 (7A6 antigen), a mitochondrial marker of apoptosis [12] was used to evaluate apoptosis by flow cytometry, as described [11].

\subsection{RhoA activation assay}

GTP-loaded RhoA levels were assessed using the Active GTPase Pull down kit (Thermo Scientific, Courtaboeuf, Fr) according to the manufacturer's instructions. Briefly, RhoA-GTP was collected using GST-Rhotekin RBD. The eluted proteins were separated by $12 \%$ SDS-PAGE, transferred to Immobilon-P membrane (Millipore, Molsheim, Fr) and detected with specific anti-RhoA antibody. Total RhoA was detected on total cell lysates. Immunoreactive bands were visualized by enhanced chemiluminescence (ECL) (Roche, Meylan, Fr).

\subsection{Generation of RhoA knockdown HMEC-1 cells}

The RhoA mRNA target sequence was GAAGUCAAGCAUUUCUGUC (RefSeq Accession Number NM_01664.2) [13]. An irrelevant scramble (scr) sequence was used as control. Short hairpin oligonucleotides sh-RhoA and sh-scr were subcloned into the lentiviral vector pFG12 [14] which allows expression of both green fluorescent protein (GFP) and target-specific shRNAs. These constructs were used for lentivirus production, as described in the ViraPower Lentiviral Expression System manual (Invitrogen, Courtaboeuf, Fr). HMEC-1 transduced either with lenti-sh-RhoA or lenti-sh-scr viral particles, were $>90 \%$ GFP-positive as estimated by flow cytometry 7 days post-infection. Transduced HMEC- 1 were used for experiments 3-4 passages post-infection. RhoA protein expression was evaluated on NP40 1\% total cell lysates by Western blot $72 \mathrm{~h}$ postinfection.

\subsection{Immunofluorescence studies}

HMEC-1 were plated on glass coverslips, serum-starved overnight and irradiated. Cells were fixed with $4 \%$ paraformaldehyde (PFA) 10 min post-IR, washed and permeabilized with $0.1 \%$ Triton $\mathrm{X}-100$ for $10 \mathrm{~min}$. After blocking in 5\% serum/PBS, cells were stained with TRITC-phalloidin to visualize F-actin and/or primary antibodies for $1 \mathrm{~h}$ at room temperature and labeled with secondary antibodies for $45 \mathrm{~min}$. Confocal microscopy was performed (Leica TCS SP, Leica Microsystems, Rueil-Malmaison, Fr). Images were processed using Metamorph software (Roper Scientific, Evry, Fr).

\subsection{Cell adhesion assay}

Adhesion assays were performed as described [15]. Briefly, cells were plated in low-serum MCDB131 medium for $2 \mathrm{~h}$ at $37^{\circ} \mathrm{C}$ in $5 \%$ $\mathrm{CO}_{2}$, then pre-treated with vehicle or $10 \mu \mathrm{M}$ Y-27632 for $30 \mathrm{~min}$ before IR. Non-adherent cells were removed $10 \mathrm{~min}$ post-IR by washing. Adherent cells were fixed, stained with $0.1 \%$ Crystal Violet solution and lysed in $0.1 \mathrm{M}$ citrate/ethanol (50/50). Absorbance was measured at $540 \mathrm{~nm}$.

\subsection{Cell migration assay}

sh-scr and sh-RhoA HMEC-1 were seeded in 12-well plates and grown until confluence before IR. The cell monolayer was then injured with a pipette tip to produce a denuded area. The culture medium was changed to eliminate detached and damaged cells. Cell migration was followed using time-lapse video microscopy (Leica DMI6000B) equipped with epifluorescence microscopy and a temperature-controlled stage $\left(37^{\circ} \mathrm{C}\right)$ under $5 \% \mathrm{CO}_{2}$. GFP expression by lentivirally-transduced HMEC- 1 allowed tracking of live infected cells at $10 \mathrm{X}$ magnification every $10 \mathrm{~min}$ for a total period of $18 \mathrm{~h}$ with $488 \mathrm{~nm}$ laser illumination (HQ2 CoolSnap camera, Roper Scientific). Image processing and data analysis were performed using Metamorph software.

\subsection{Statistical analysis}

All values were reported as mean \pm SEM, as indicated. Statistical significance was evaluated $p<0.05$, unless otherwise indicated, using Student's $t$ test (GraphPad Instat3 software, La Jolla, USA).

\section{Results}

3.1. RhoA/ROCK pathway is involved in rapid reorganization of actin cytoskeleton induced by IR

To determine the significance of RhoA in radiation response of EC, we first analyzed RhoA activation by mean of its GTP-loading in 15 Gy-irradiated HMEC-1. As shown in Fig. 1A, we detected a very low level of GTP-RhoA in non-irradiated ( 0 Gy) cells, which increased significantly within $10 \mathrm{~min}$ following IR, indicating that RhoA was rapidly activated in EC exposed to $15 \mathrm{~Gy}$. Next, actin cytoskeleton remodeling was assessed, as the main role of RhoA via its effector ROCK described in mammalian cells is the formation of stress fibers [4]. Non-irradiated HMEC-1 presented sparse stress fibers spanning the cell body whereas in $15 \mathrm{~Gy}$-irradiated cells, a rapid and profound reorganization of the actin cytoskeleton was observed, characterized by a significant increase in thick stress fibers. Inhibition of ROCK by pre-treatment of EC with inhibitor Y-27632 led to a complete disappearance of F-actin bundles in non-irradiated cells and completely abrogated $15 \mathrm{~Gy}$-induced increase of stress fibers, confirming the RhoA/ROCK 
A

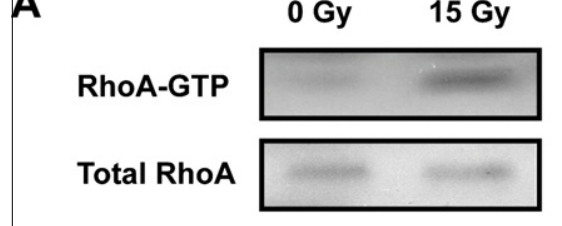

C

D

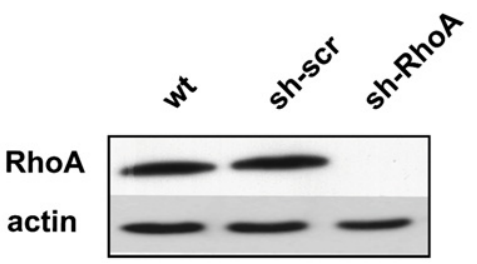

sh-scr

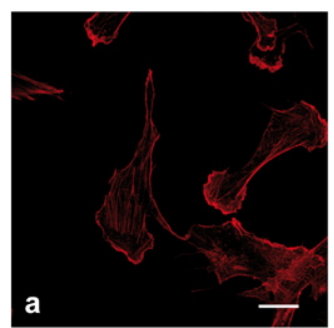

o Gy

sh-RhoA
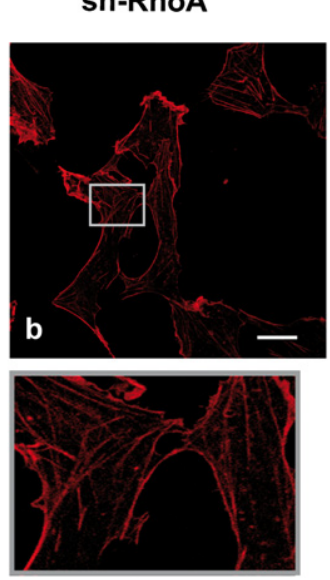

B
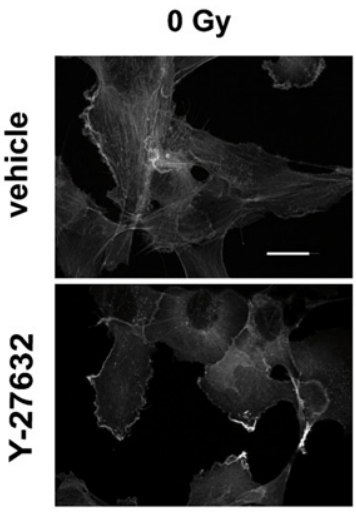

15 Gy

sh-scr
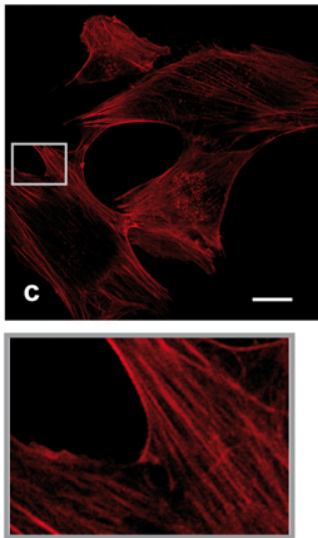

15 Gy

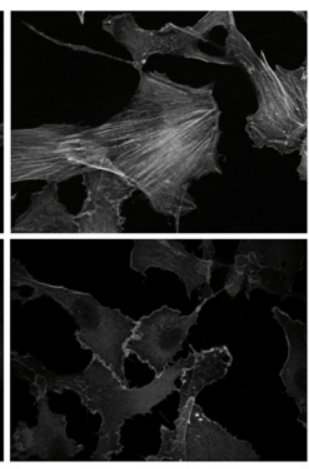

sh-RhoA
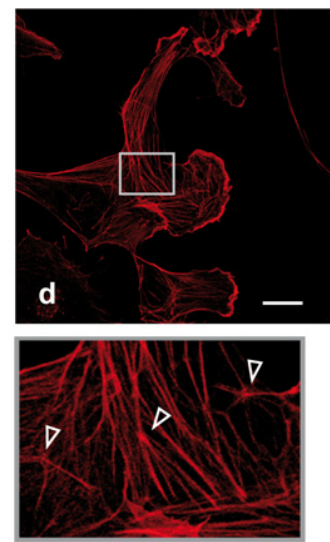

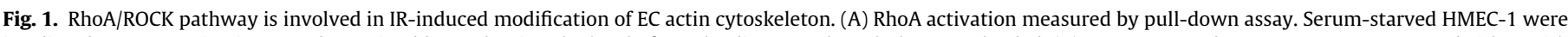

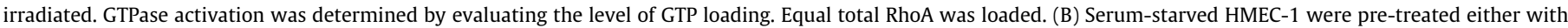

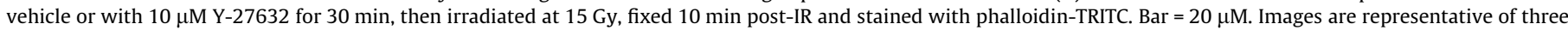

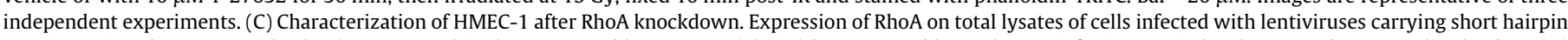

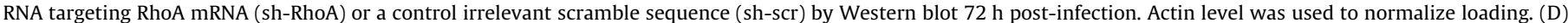

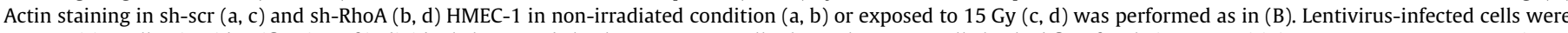

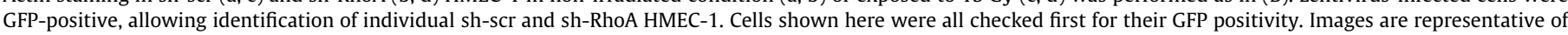

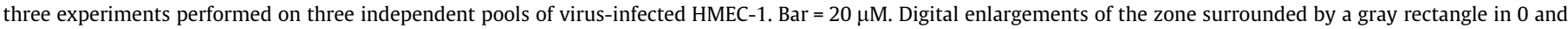
$15 \mathrm{~Gy}$ conditions are shown, $(\triangle)$ indicate actin nodes.

involvement in this extensive actin remodeling (Fig. 1B). In order to characterize more precisely the implication of RhoA in EC response to IR, RNA interference was used to knockdown RhoA expression in HMEC-1. RhoA protein expression was totally suppressed in HMEC-1 infected with sh-RhoA, but remained unaffected in sh-scr cells (Fig. 1C). RhoA silencing has no effect on the growth characteristics of HMEC-1 in basal conditions (data not shown). Analyzing the actin cytoskeleton (Fig. 1D) of nonirradiated sh-RhoA HMEC-1 revealed no obvious modifications in F-actin organization compared to non-irradiated sh-scr HMEC-1 ( $a$ and $b$ ), with presence of small numbers of stress fibers spanning the cell body. In sh-scr HMEC-1 irradiated at $15 \mathrm{~Gy}$, we observed a massive increase in stress fibers with thick actin cables crossing the cell body ( $\mathrm{a}$ and $\mathrm{c}$ ), as previously shown in HMEC-1 exposed to $15 \mathrm{~Gy}$ (Fig. 1A). However, in sh-RhoA HMEC-1, IR led to a totally different actin organization profile, with thinner bundles connected to actin nodes (d), different from fully well organized thick stress fibers. These results indicated that IR induced acute actin cytoskeleton remodeling in EC, dependent on the RhoA/ROCK pathway and characterized by formation of stress fibers.

\subsection{RhoA/ROCK pathway is not involved in IR-induced cell death}

Irradiation of HMEC-1 led to a significant 2-fold increase in apoptosis (Fig. 2A) as previously reported [11]. Pre-treatment with Y-27632 did not modify the increased cell death in irradiated HMEC-1. Both irradiated sh-scr HMEC-1 and sh-RhoA HMEC-1 displayed a $44 \%$ increase in apoptosis compared to their non-irradiated counterparts (Fig. 2B). These results proved that apoptotic fate of EC exposed to IR did not require RhoA/ROCK.

\subsection{RhoA/ROCK pathway participates to IR-induced increase of adhesion to fibronectin}

Adhesion to fibronectin, a cellular function known to be linked to actin cytoskeleton dynamics [16], was investigated in irradiated HMEC-1. Specific adhesion to fibronectin was enhanced by 2-fold following IR (Fig. 3A), both in wt (left panel) and sh-scr cells (right panel). In contrast, this IR-increased adhesion was fully abolished in Y-27632 pre-treated cells (left panel) or in invalidated sh-RhoA HMEC-1 (right panel). Focal adhesions are specialized sites containing a complex meshwork of proteins 

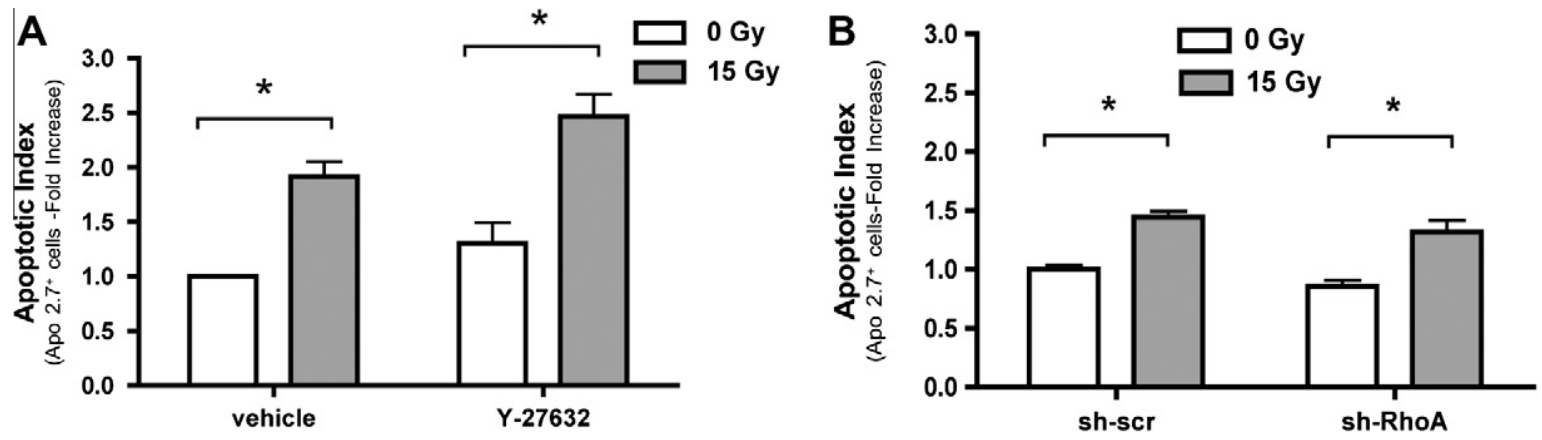

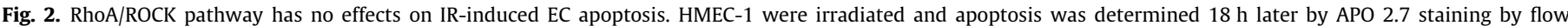

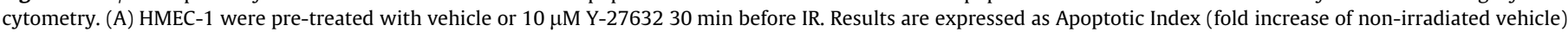

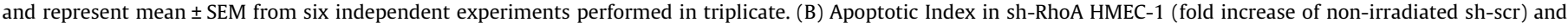
represent mean \pm SEM from three independent experiments performed in triplicate. ${ }^{*} p<0.05$ (Student's $t$-test).
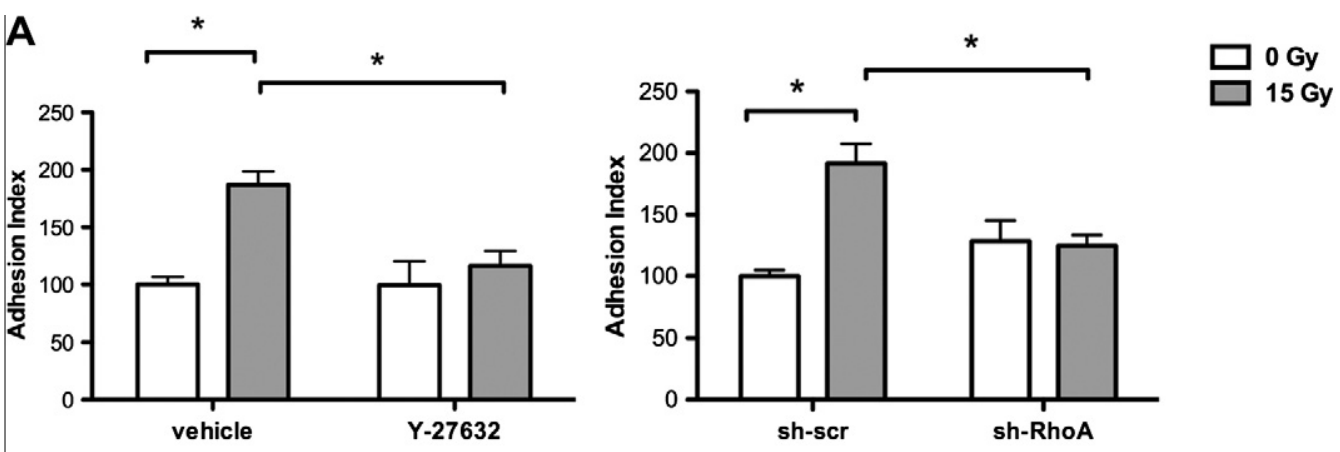

B

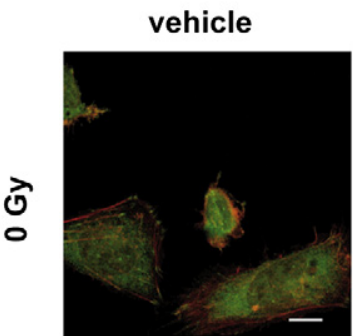

Y-27632
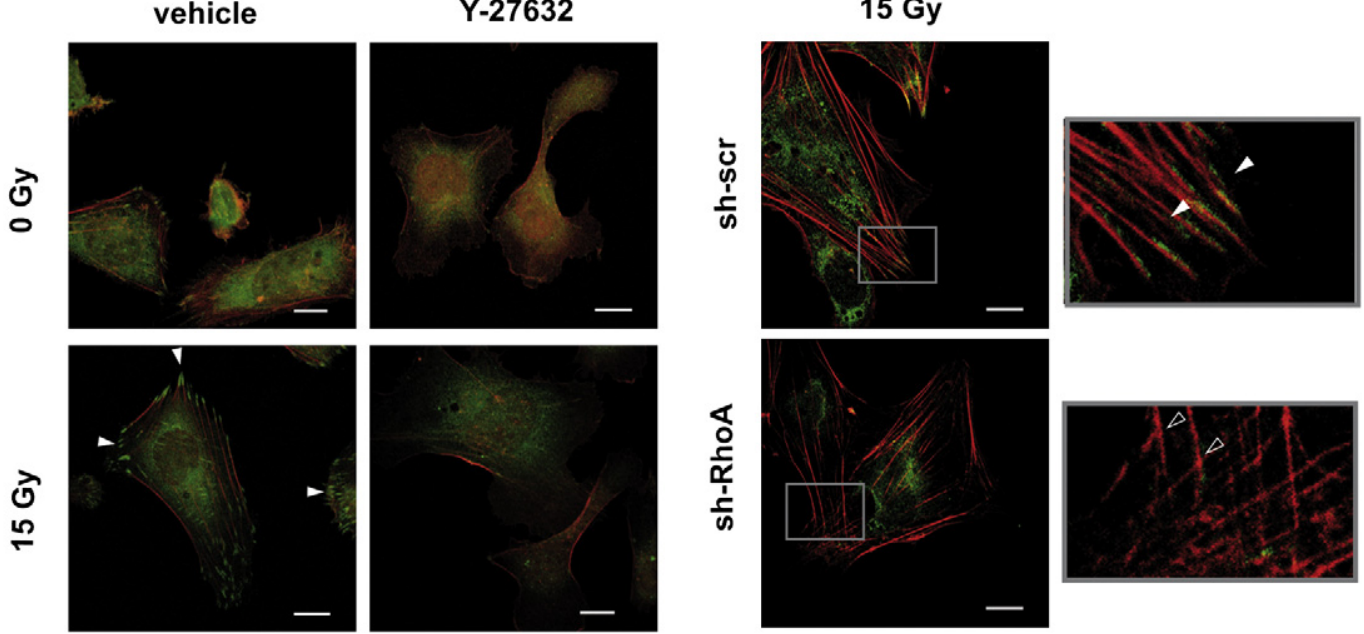

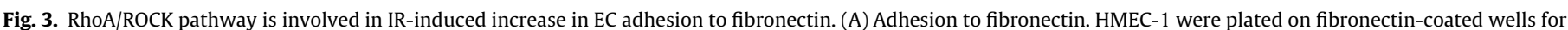

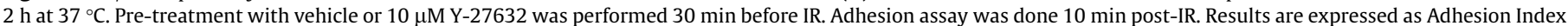

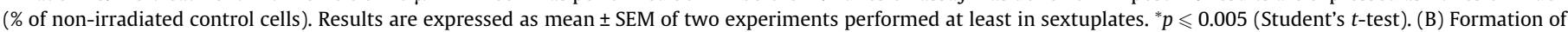

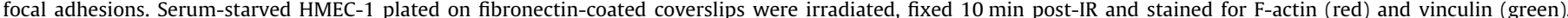

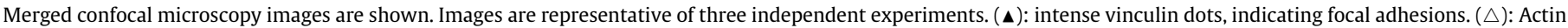

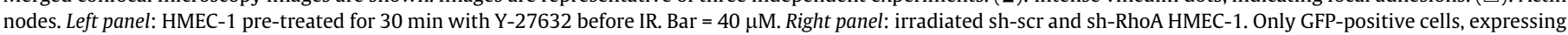
sh-scr or sh-RhoA, were analyzed. Digital enlargement of the zone outlined in sh-scr or sh-RhoA cells is shown. Bar $=20 \mu \mathrm{M}$.

included vinculin and favoring interactions between extracellular matrix (ECM)-linked integrins and the actin cytoskeleton [16]. As their formation reflects cellular adhesion, detection of these structures by vinculin immunostaining was conducted in HMEC-1 plated on fibronectin (Fig. 3B). In non-irradiated EC, vinculin staining was mainly diffuse throughout the cytoplasm, with a few small dots on the cell periphery. In contrast, in irradiated cells, accumulation of intense elongated dots at the cell periphery revealed the presence of mature focal adhesions (left panel). As in Fig. 1A, irradiated HMEC-1 stained for F-actin showed increased stress fibers (Fig. 3B, left panel) and focal adhesions were localized at the termini of these cytoskeletal structures. Pre-treatment of HMEC-1 with Y-27632 led to a complete absence of vinculin dots with or without IR exposure. As shown in Fig. 1B, Y-27632 also abolished IR-induced increase in stress fibers formation in fibronectin-plated HMEC-1. In knockdown experiments (Fig. 3B, right panel), IR induced the formation of focal adhesions in sh-scr HMEC-1 whereas in 

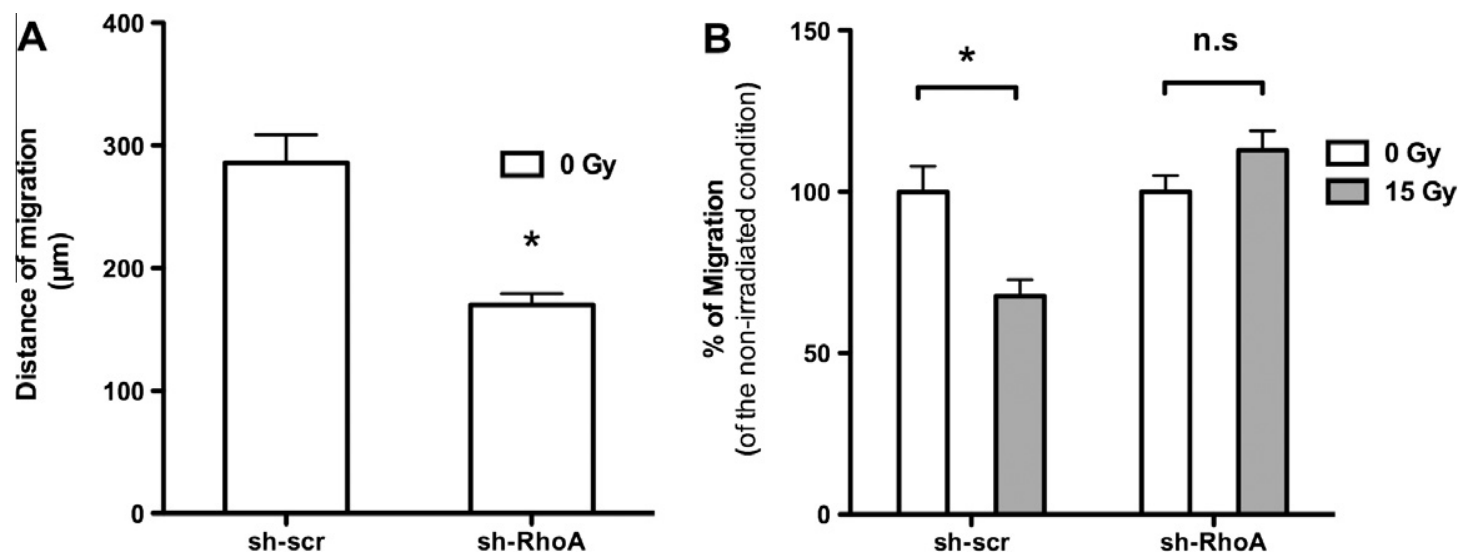

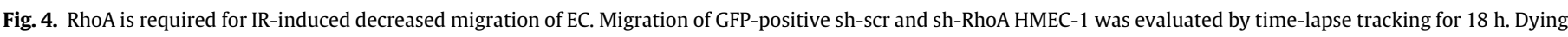

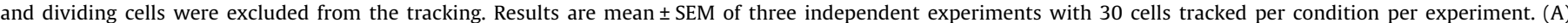

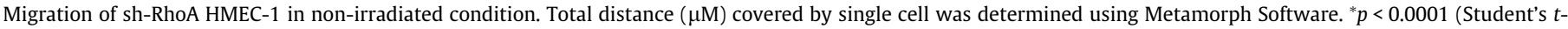
test). (B) Migration of sh-scr and sh-RhoA expressed as percentage of the non-irradiated condition. ${ }^{*} p<0.0005$, n.s: non significant (Student's $t$-test).

irradiated sh-RhoA HMEC-1, vinculin staining was weak and diffuse throughout the cytoplasm. Importantly, no vinculin dots were seen, indicating an absence of mature focal adhesions in these cells. Irradiated sh-RhoA HMEC-1 formed short thin bundles connected to actin nodes, as previously illustrated in Fig. 1D.

Altogether, these results indicated that IR-induced increased adhesion of EC was entirely dependent on an efficient RhoA/ROCK pathway, which promoted formation of focal adhesions.

\subsection{IR-induced inhibition of EC migration requires RhoA activity}

We next investigated migration of irradiated HMEC- 1 by filming the recolonization of a denuded scratch of a confluent EC monolayer (Fig. 4). In order to evaluate only migratory processes, neither dividing nor dying cells were tracked. Invalidated sh-RhoA EC in non-irradiated condition (Fig. 4A) migrated on a $40 \%$ shorter distance than sh-scr cells. Upon IR exposure, migration of sh-scr HMEC- 1 was decreased by $32 \%$, compared to non-irradiated cells (Fig. 4B). In contrast, IR-induced inhibition of migration was not observed in sh-RhoA HMEC-1. Thus, IR partially reduced HMEC-1 migration, but this inhibition was no longer observable in the absence of RhoA. These data indicated that RhoA, although required for basal migration, participated also to IR-induced inhibition of endothelial migration.

\section{Discussion}

Damage to capillary blood vessels is one of the most common effects of radiation and injury to the vascular endothelium is a key factor in early and delayed radiation toxicity in tissues. Despite improved characterization of molecular signaling pathways mobilized for acute cellular responses to radiation, the role of membrane-linked GTPase RhoA was underinvestigated. The present work now identifies RhoA as an important actor in endothelial early responses to IR, in particular in actin cytoskeleton remodeling, adhesion to fibronectin and migration. Rapid activation of RhoA and stress fibers formation was previously described in primary human dermal microvascular EC upon IR exposure [3]. As perturbation of cell morphology was one of the obvious early hallmarks of immediate effects of IR on the endothelial compartment $[17,18]$, our data together with recent work [3] strongly support an essential role for RhoA in immediate modifications of the cellular architecture in response to high dose of IR in microvascular EC.
High dose of IR are known to engage ECs toward cell death, mainly through ceramide-mediated apoptosis both in vitro $[11,19]$ and in vivo $[20,21]$. Despite extensive investigations of the involvement of RhoA in the apoptotic process, the current understanding is that RhoA can lead either to pro-apoptotic or pro-survival signals, depending on cell type and/or apoptotic stimulus [22], particularly in the endothelial compartment [6,7]. Thus, in radiation-mediated apoptosis of EC, RhoA GTPases do not appear as mandatory signaling molecules. As already mentioned, the endothelium has also the capacity to react to IR stress by acquiring an activated phenotype linked to changes in morphology, adhesion, permeability and secretion [2]. This study demonstrated that IR affected adhesion to fibronectin and endothelial migration and these events were dependent on RhoA/ROCK signaling. RhoA GTPases are known to regulate cellular adhesion partly through the regulation of focal adhesion assembly, as a result of mechanical force generated by actin cytoskeleton-derived stress fibers [23]. Indeed, our results highlighted the role of RhoA/ROCK in IR-increased adhesion, as RhoA-dependent stress fiber formation was required to produce focal adhesions. Filming irradiated HMEC-1 revealed reduced migration in living and non-dividing cells, also consistent with increased adhesion. This confirmed the inhibitory effect of IR on microvessel migration, previously shown on primary microvascular EC and quiescent macrovessels [24,25]. Furthermore, our results showed that the fraction of EC not committed to cell death displayed a lower ability to recolonize denuded zones and this would make them less efficient in restoring monolayer integrity following IR-induced damage. This study also demonstrated that RhoA GTPase affected differently EC migration, generating promigratory signals in non-irradiated conditions, but required in the anti-migratory response observed in irradiated EC as nonirradiated and irradiated sh-RhoA HMEC-1 showed equivalent levels of migration. The balance between migration and adhesion requires fine tuning with both spatial and temporal coordination of the actin cytoskeleton and extracellular adhesion [26]. The role of RhoA GTPases in these processes remains complex but the level of activation of these proteins appears to determine the response: RhoA have been described as contributing to migration, while at the same time high RhoA activity strongly inhibits migration by promoting very efficient adhesion [26,27]. In our model, in nonirradiated EC, RhoA, which presented a low level of activation RhoA is necessary for migration whereas EC migration was impaired in irradiated conditions where RhoA is strongly activated.

Characterization of RhoA/ROCK pathway as rapidly recruited in endothelial cells in response to radiation raised the issue of how 
radiation could lead to membrane-linked protein RhoA activation. Massive production of reactive oxygen species (ROS) is the first intracellular radiochemical event after radiation exposure and could possibly lead to RhoA activation, as this protein was very recently identified as a ROS/RNS target [28]. Another potential pathway leading to RhoA activation could be the sphingolipid ceramide generated by IR in the plasma membrane [29], already identified in in vitro studies as activating RhoA in EC [30] and described as an important messenger in transduction of cellular stress [31].

Membrane signaling GTPases RhoA are now identified in EC as important mediator of immediate effects of IR, leading to increased permeability [3] and also to enforced adhesion and decreased migration. These altered functions conferred to EC an aberrant activated phenotype which would conduct to vascular dysfunctions promoting tissue inflammation, rupture of capillaries and eventually to more delayed damages relying on premature EC senescence. Indeed, pro-adhesive with impaired migratory flatted phenotype was recently described as hallmark of senescent irradiated endothelium [24]. Nowadays, protecting normal tissue of immediate damages of radiation is a major goal. Inhibiting the RhoA/ROCK pathway has already been shown to reduce IR-induced intestinal toxicity [32]. Therefore, targeting the RhoA/ROCK pathway by pharmacological agents could be useful to limit radiation-induced endothelial dysfunctions for both clinical management of accidental or radiotherapy toxicity on normal tissues.

\section{Acknowledgments}

The authors are grateful to Philippe Hulin and Monique Clément from the MicroPICell - Cellular Imaging Core Facility of the IRT-UN for help and advice. We thank Stéphane Supiot and Sylvie Hermouet for critical reading of the manuscript. This work was financially supported by Electricite de France (EDF) and La Ligue contre le Cancer.

\section{References}

[1] D.G. Baker, R.J. Krochak, The response of the microvascular system to radiation: a review, Cancer Invest. 7 (1989) 287-294.

[2] M.H. Gaugler, A unifying system: does the vascular endothelium have a role to play in multi-organ failure following radiation exposure?, BJR Suppl. 27 (2005) 100-105.

[3] D. Gabrys, O. Greco, G. Patel, K.M. Prise, G.M. Tozer, C. Kanthou, Radiation effects on the cytoskeleton of endothelial cells and endothelial monolayer permeability, Int. J. Radiat. Oncol. Biol. Phys. 69 (2007) 1553-1562.

[4] S. Etienne-Manneville, A. Hall, Rho GTPases in cell biology, Nature 420 (2002) 629-635.

[5] G.P. van Nieuw Amerongen, V.W. van Hinsbergh, Cytoskeletal effects of rholike small guanine nucleotide-binding proteins in the vascular system, Arterioscler. Thromb. Vasc. Biol. 21 (2001) 300-311.

[6] X. Li, L. Liu, J.C. Tupper, D.D. Bannerman, R.K. Winn, S.M. Sebti, A.D. Hamilton, J.M. Harlan, Inhibition of protein geranylgeranylation and RhoA/RhoA kinase pathway induces apoptosis in human endothelial cells, J. Biol. Chem. 277 (2002) 15309-15316.

[7] I. Petrache, K. Birukov, A.L. Zaiman, M.T. Crow, H. Deng, R. Wadgaonkar, L.H. Romer, J.G. Garcia, Caspase-dependent cleavage of myosin light chain kinase (MLCK) is involved in TNF-alpha-mediated bovine pulmonary endothelial cell apoptosis, FASEB J. 17 (2003) 407-416.

[8] N. McLaughlin, B. Annabi, M. Bouzeghrane, A. Temme, J.P. Bahary, R. Moumdjian, R. Beliveau, The survivin-mediated radioresistant phenotype of glioblastomas is regulated by RhoA and inhibited by the green tea polyphenol (-)-epigallocatechin-3-gallate, Brain Res. 1071 (2006) 1-9.
[9] T. Nubel, W. Dippold, B. Kaina, G. Fritz, Ionizing radiation-induced E-selectin gene expression and tumor cell adhesion is inhibited by lovastatin and alltrans retinoic acid, Carcinogenesis 25 (2004) 1335-1344.

[10] T. Ishizaki, M. Uehata, I. Tamechika, J. Keel, K. Nonomura, M. Maekawa, S. Narumiya, Pharmacological properties of Y-27632, a specific inhibitor of rhoassociated kinases, Mol. Pharmacol. 57 (2000) 976-983.

[11] S. Bonnaud, C. Niaudet, G. Pottier, M.H. Gaugler, J. Millour, J. Barbet, L. Sabatier, F. Paris, Sphingosine-1-phosphate protects proliferating endothelial cells from ceramide-induced apoptosis but not from DNA damage-induced mitotic death, Cancer Res. 67 (2007) 1803-1811.

[12] C. Zhang, Z. Ao, A. Seth, S.F. Schlossman, A mitochondrial membrane protein defined by a novel monoclonal antibody is preferentially detected in apoptotic cells, J. Immunol. 157 (1996) 3980-3987.

[13] C. Deroanne, V. Vouret-Craviari, B. Wang, J. Pouyssegur, EphrinA1 inactivates integrin-mediated vascular smooth muscle cell spreading via the Rac/PAK pathway, J. Cell Sci. 116 (2003) 1367-1376.

[14] X.F. Qin, D.S. An, I.S. Chen, D. Baltimore, Inhibiting HIV-1 infection in human T cells by lentiviral-mediated delivery of small interfering RNA against CCR5, Proc. Natl. Acad. Sci. USA 100 (2003) 183-188.

[15] M.J. Humphries, Cell adhesion assays, Methods Mol. Biol. 139 (2000) 279-285.

[16] E. Zamir, B. Geiger, Molecular complexity and dynamics of cell-matrix adhesions, J. Cell Sci. 114 (2001) 3583-3590.

[17] M. Friedman, U.S. Ryan, W.C. Davenport, E.L. Chaney, D.L. Strickland, L. Kwock, Reversible alterations in cultured pulmonary artery endothelial cell monolayer morphology and albumin permeability induced by ionizing radiation, J. Cell. Physiol. 129 (1986) 237-249.

[18] J.M. Onoda, S.S. Kantak, C.A. Diglio, Radiation induced endothelial cell retraction in vitro: correlation with acute pulmonary edema, Pathol. Oncol. Res. 5 (1999) 49-55.

[19] A. Haimovitz-Friedman, C.C. Kan, D. Ehleiter, R.S. Persaud, M. McLoughlin, Z. Fuks, R.N. Kolesnick, Ionizing radiation acts on cellular membranes to generate ceramide and initiate apoptosis, J. Exp. Med. 180 (1994) 525-535.

[20] M. Garcia-Barros, F. Paris, C. Cordon-Cardo, D. Lyden, S. Rafii, A. HaimovitzFriedman, Z. Fuks, R. Kolesnick, Tumor response to radiotherapy regulated by endothelial cell apoptosis, Science 300 (2003) 1155-1159.

[21] F. Paris, Z. Fuks, A. Kang, P. Capodieci, G. Juan, D. Ehleiter, A. HaimovitzFriedman, C. Cordon-Cardo, R. Kolesnick, Endothelial apoptosis as the primary lesion initiating intestinal radiation damage in mice, Science 293 (2001) 293297.

[22] J. Shi, L. Wei, Rho kinase in the regulation of cell death and survival, Arch. Immunol. Ther. Exp. (Warsz) 55 (2007) 61-75.

[23] A.D. Dubash, M.M. Menold, T. Samson, E. Boulter, R. Garcia-Mata, R. Doughman, K. Burridge, Chapter 1. Focal adhesions: new angles on an old structure, Int. Rev. Cell. Mol. Biol. 277 (2009) 1-65.

[24] N. Imaizumi, Y. Monnier, M. Hegi, R.O. Mirimanoff, C. Ruegg, Radiotherapy suppresses angiogenesis in mice through TGF-betaRI/ALK5-dependent inhibition of endothelial cell sprouting. PLoS ONE 5 (2010) e11084.

[25] M. Scharpfenecker, J.J. Kruse, D. Sprong, N.S. Russell, P. Ten Dijke, F.A. Stewart, Ionizing radiation shifts the PAI-1/ID-1 balance and activates notch signaling in endothelial cells, Int. J. Radiat. Oncol. Biol. Phys. 73 (2009) 506-513.

[26] A.J. Ridley, M.A. Schwartz, K. Burridge, R.A. Firtel, M.H. Ginsberg, G. Borisy, J.T. Parsons, A.R. Horwitz, Cell migration: integrating signals from front to back, Science 302 (2003) 1704-1709.

[27] E.A. Cox, S.K. Sastry, A. Huttenlocher, Integrin-mediated adhesion regulates cell polarity and membrane protrusion through the Rho family of GTPases, Mol. Biol. Cell 12 (2001) 265-277.

[28] A. Aghajanian, E.S. Wittchen, S.L. Campbell, K. Burridge, Direct activation of RhoA by reactive oxygen species requires a redox-sensitive motif, PLoS ONE 4 (2009) e8045.

[29] J.P. Truman, M. Garcia-Barros, M. Kaag, D. Hambardzumyan, B. Stancevic, M. Chan, Z. Fuks, R. Kolesnick, A. Haimovitz-Friedman, Endothelial membrane remodeling is obligate for anti-angiogenic radiosensitization during tumor radiosurgery, PLoS ONE 5 (2010) e12310.

[30] N. Gupta, E. Nodzenski, N.N. Khodarev, J. Yu, L. Khorasani, M.A. Beckett, D.W. Kufe, R.R. Weichselbaum, Angiostatin effects on endothelial cells mediated by ceramide and RhoA, EMBO Rep. 2 (2001) 536-540.

[31] B. Stancevic, R. Kolesnick, Ceramide-rich platforms in transmembrane signaling, FEBS Lett. 584 (2010) 1728-1740.

[32] C. Bourgier, V. Haydont, F. Milliat, A. Francois, V. Holler, P. Lasser, J. Bourhis, D. Mathe, M.C. Vozenin-Brotons, Inhibition of Rho kinase modulates radiation induced fibrogenic phenotype in intestinal smooth muscle cells through alteration of the cytoskeleton and connective tissue growth factor expression, Gut 54 (2005) 336-343. 